\title{
Diabetic neuropathy in the gut: pathogenesis and diagnosis
}

\author{
Fernando Azpiroz ${ }^{1,2,3}$ - Carolina Malagelada ${ }^{1,2,3}$
}

Received: 15 October 2015 / Accepted: 4 November 2015 / Published online: 7 December 2015

(C) Springer-Verlag Berlin Heidelberg 2015

\begin{abstract}
The activity of the digestive tract is usually regulated to match its content: physiological stimuli in the gut induce modulatory reflexes that control digestive function so that digestion is normally not perceived. However, under certain circumstances, digestive stimuli may activate sensory afferents and give rise to conscious sensations. Both reflex and sensory signals are modulated by a balance of excitatory and inhibitory mechanisms. Patients with diabetes may develop a neuropathy affecting the control of gastric and/or intestinal motor function and the sensory innervation as well. During fasting the stomach is contracted and relaxes to accommodate a meal. After ingestion the stomach progressively recontracts and this contraction gently produces gastric emptying. Impairment of excitatory pathways affects the contraction of the stomach, which may result in delayed gastric emptying and vomiting of retained food. Conversely, alteration of the inhibitory neural pathways results in impaired relaxation of the stomach in response to a meal; in this case increased wall tension may produce early satiation, fullness and nausea. Diabetic neuropathy may distort the control of intestinal motility, which can lead to diverse symptoms such as diarrhoea, constipation, intestinal distension and abdominal pain.
\end{abstract}

Fernando Azpiroz

azpiroz.fernando@gmail.com

1 Digestive System Research Unit, Hospital General Vall d'Hebron, 08035 Barcelona, Spain

2 Centro de Investigación Biomédica en Red de Enfermedades Hepáticas y Digestivas (Ciberehd), Spain

http://www.ciberehd.org

3 Departament de Medicina, Universitat Autònoma de Barcelona, Bellaterra, Cerdanyola del Vallès, Spain
Neuropathy in diabetes may also affect the sensory nerves of the gut, and depending on which pathways are involved, perception may be increased or reduced. In summary, in patients with diabetic neuropathy, disorders of gut motor function are associated with sensory abnormalities, and the combination of impaired pathways determines the clinical consequences. This review summarises a presentation given at the 'Diagnosis and treatment of autonomic diabetic neuropathy in the gut' symposium at the 2015 annual meeting of the EASD. It is accompanied by another mini-review on a topic from this symposium (by Hans Törnblom, DOI: 10.1007/s00125-0153829-9) and a commentary by the Session Chair, Péter Kempler (DOI: 10.1007/s00125-015-3826-y).

Keywords Autonomic neuropathy $\cdot$ Diabetes $\cdot$ Enteric neuropathy · Functional gut disorders · Gastric motility · Gut reflexes $\cdot$ Intestinal motility $\cdot$ Sensory pathways

\section{Introduction}

Under normal conditions, digestion is not perceived because the gut adapts its activity to its content: physiological stimuli in the gut induce neurohumoral reflexes that control digestive function. However, under certain circumstances digestive stimuli may activate sensory afferents and give rise to conscious sensations.

Patients with diabetes may develop nerve damage (neuropathy) affecting the nerves that control gut function and perception. Neuropathy of the gut can cause diverse dysfunctions that may range from mild, even unperceived by the patient, to very severe and incapacitating. These dysfunctions may affect the stomach and the intestine, as well as other abdominal organs [1] (Fig. 1). 
Fig. 1 Diabetic neuropathy may affect reflex and sensory pathways controlling gastric and intestinal function. Both sensory and reflex pathways are modulated by a series of excitatory and inhibitory mechanisms. Clinical manifestations depend on the pathways, modulatory mechanisms and areas of the gut affected

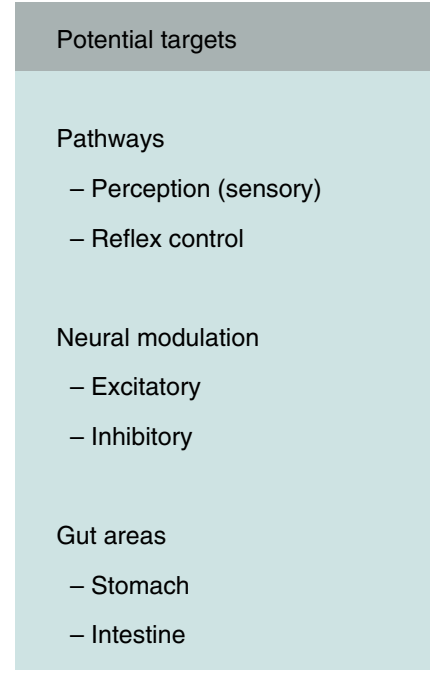

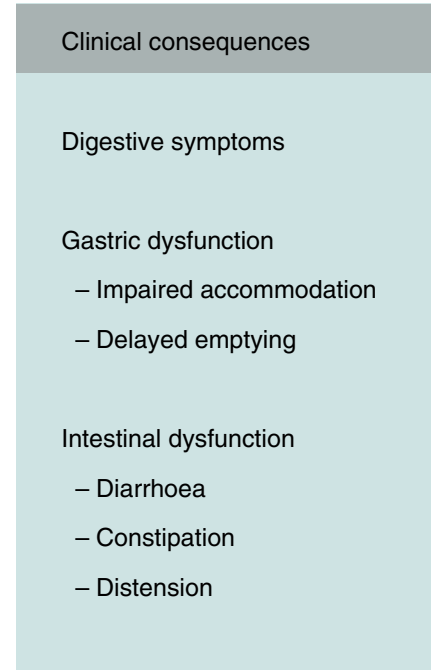

\section{Gastrointestinal neural pathways}

Similar to other parts of the gastrointestinal tract, the myenteric plexus is found between the longitudinal and circular muscle layers of the stomach. Intrinsic neurons are organised in ganglia, the number of which increases towards the distal antrum. Although these neurons are modulated by vagal (parasympathetic) and splanchnic (sympathetic) extrinsic nerves, the gastric myenteric plexus has functional autonomy.

The vagus nerves play an essential role in the control of gastric motility. Vagal cell bodies are located in the nodose ganglion and have predominantly afferent fibres (80-90\%) that convey information to neurons in the nucleus of the solitary tract. The incoming information is processed and activates efferent vagal motor neurons in the dorsal motor nucleus or in the nucleus ambiguus. These so-called vagovagal reflexes control both gastric emptying and intestinal transit of chyme.

Sympathetic (or splanchnic) innervation of the stomach derives from spinal segments T6-T9. By inhibiting presynaptic acetylcholine release from the myenteric plexus, these nerves mainly exert an inhibitory function via sympathetic reflexes relaying at prevertebral ganglia. Perception of gastric stimuli is driven by splanchnic-sympathetic-spinal nerves, and vagal fibres may only play a modulatory role [2].

\section{Involvement of gastric control pathways}

The stomach accommodates a rapidly ingested, heterogeneous meal, and delivers into the small bowel homogenised chyme at a rate adapted to the processing capability of the intestine. The stomach is emptied by a finely modulated, sustained contraction of the proximal part of the stomach, i.e. the corpus and fundus regions. This tonic contraction is known as gastric tone. Grinding of solids occurs in the distal part, i.e. the antrum. The pyloric region is able to discriminate between solids and liquids and retains in the antrum particles larger than $1 \mathrm{~mm}$, which need to be ground before they can be evacuated into the duodenum.

In the fasted state the stomach is virtually empty and is contracted. During ingestion the stomach relaxes to accommodate the meal without increasing the tension of its walls. Once ingestion has finished, the stomach progressively contracts, i.e. regains tone, and this tonic contraction gently forces intragastric chyme distally, loading the antral pump and facilitating gastric emptying. Liquid chyme is squeezed through the pyloric sphincter, which determines the final gastric outflow, while solid material is retained and ground in the antrum by phasic contractions. This process is precisely regulated so that the delivery of nutrients is matched to the capacity of the intestine to process them.

\section{Impaired gastric contraction}

Muscular activity of the stomach depends on the balance of excitatory and inhibitory neural pathways. Alteration of the excitatory pathways impairs the contractile ability of the stomach - a condition known as gastroparesis - and this results in delayed gastric emptying (Fig. 2). In severe cases the stomach becomes flaccid and unable to empty its contents, leading to nausea and vomiting of retained food. This condition is particularly problematic in patients with diabetes because it interferes with the absorption of sugars and medication and therefore contributes to poor control of glycaemia [3].

\section{Impaired gastric relaxation}

Alteration of the inhibitory neural pathways results in impaired relaxation of the stomach in response to a meal. If this occurs, meal ingestion increases the tension of the wall of 


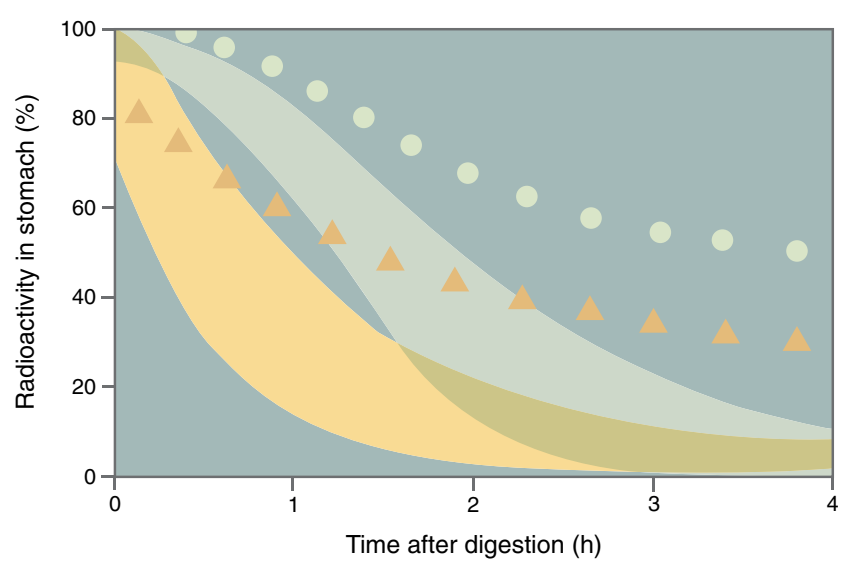

Fig. 2 Example of diabetic gastroparesis. Solid and liquid components of a meal are labelled with radioactive isotopes and gastric emptying is measured as gastric clearance in sequential scans by scintigraphy. Shaded areas represent the normal range for liquid (orange) and solid (light blue) emptying. Under normal conditions the emptying of solids is slower, because solid particles require a preliminary grinding process. The figure shows the emptying of solids (light blue circles) and liquids (orange triangles) in a patient with diabetic gastroparesis; note the delayed emptying, indicated by the values outside the normal range

the stomach. Gastric wall tension receptors are linked to afferent nerve pathways that send the afferent signal up to the brain cortex, inducing conscious perception. Increased wall tension causes early satiation, inability to finish a meal, fullness and nausea [4]. In contrast to gastroparesis, gastric emptying is normal.

\section{Measurement of gastric emptying}

Gastric emptying can be evaluated by different techniques.

Radionuclide gastric emptying test Radioscintigraphy measurement of the gastric emptying rate remains the gold standard for quantitative assessment of the emptying function of the stomach. To perform this test, patients are administered a standarised meal in which one component (or several) has been labelled with a radioisotopic $\gamma$-emitting marker. An external $\gamma$-camera quantifies the radioactivity in the area of the stomach in sequential images over $4 \mathrm{~h}$. The rate of disappearance of the radioisotope corresponds to the gastric emptying rate [5]. Solids and liquids are emptied from the stomach at different rates and can be independently evaluated by labelling each component with a different radioisotope (Fig. 2).

${ }^{13} \mathrm{C}$ breath test Gastric emptying can also be evaluated indirectly by labelling the solid or liquid phase of a meal with a ${ }^{13} \mathrm{C}$-containing substrate (octanoic acid, acetic acid, glycin or spirulina). When the meal enters the intestine, the ${ }^{13} \mathrm{C}$-containing substrate is rapidly absorbed and metabolised in the liver to ${ }^{13} \mathrm{CO}_{2}$, which is then excreted through the breath. The amount of ${ }^{13} \mathrm{CO}_{2}$ in successive breath samples is directly proportional to the gastric emptying rate [6]. This test has the advantages that the label is non-radioactive and the test is simple to perform. However, the test is not well standardised, and therefore is not considered the gold standard for the diagnosis of gastroparesis.

Ultrasound Gastric ultrasound can be used to quantify gastric emptying of liquids. It is performed by consecutive measurements of the gastric antral diameter after ingestion of a liquid meal [7]. Gastric emptying is considered complete when the antral diameter returns to its basal value. Even though this tool is used for experimental purposes, it is not widely used in clinical settings because of its operator dependence.

\section{Measurement of gastric tonic contraction}

The tonic contraction of the stomach can be measured by means of a barostat, which is a computerised air pump connected via a thin tube to a bag. The bag is introduced into the stomach and the pump is programmed to maintain a constant pressure within the bag. When the stomach relaxes, the system injects air, and when the stomach contracts, air is aspirated. Hence, the volume of air within the bag reflects the degree of contraction of the stomach: at a constant pressure, a large volume reflects a gastric relaxation and a small volume represents a contraction. Patients with gastroparesis exhibit a flaccid, weak stomach with a large volume of air in the intragastric bag at relatively low intragastric pressures [8]. Gastric relaxation after a meal is normally detected as a volume increment at constant pressure, and this response is blunted in patients with impaired gastric accommodation [9].

\section{Involvement of intestinal control pathways}

Like the stomach, the activity of the intestine is determined by its content. The intestine manages its content by different types of annular contractions, which, depending on their temporo-spatial pattern, may produce segmentation/mixing or propulsion of contents. The organisation of intestinal contractile activity is still poorly understood. Diabetic neuropathy may disturb the regulation of intestinal motility and manifest as diverse symptoms such as diarrhoea, constipation, intestinal distension or abdominal pain [1]. These symptoms may present simultaneously or may alternate over time.

\section{Evaluation of intestinal function}

Intestinal manometry The current gold standard technique for the evaluation of intestinal motility is manometry. Basically, a series of pressure sensors are introduced into the intestine, conventionally mounted over a tube that can be introduced via the mouth or the anus to evaluate either the small bowel or the colon. When the intestine contracts over a sensor, the increase 
in pressure is recorded. The use of multiple sensors gives an idea of the temporo-spatial organisation of contractions, and some specific abnormal patterns have been identified in patients with intestinal neuropathy [10]. Of note, these patterns are not specific to diabetes, but also appear in other types of disorders that affect the neural pathways controlling intestinal function at any level between the gut and the central nervous system.

Intestinal manometry has several limitations. It is an invasive procedure involving discomfort for the patient, requires considerable expertise and is restricted to referral centres. As mentioned above, the evaluation of intestinal motility with manometry is exclusively based on the pattern of occlusive intestinal contractions sensed by the recording system. Manometry does not measure other signs of intestinal motor dysfunction, such as tone or transit of chyme. The consequence of this limited picture is that even in patients with significant intestinal neuropathy, most of the manometric recording is similar to that for healthy individuals and abnormal patterns appear only episodically and for brief periods of time. Moreover, intestinal manometry is relatively insensitive to less severe, or early, intestinal motor dysfunction.

Endoluminal image analysis A system has recently been developed that allows visualisation of the interior of the digestive tract by means of a small capsule that incorporates a camera with a flash. Once ingested, the capsule takes sequential pictures of the gut lumen that are transmitted to an external recorder and visualised as a video using a specific computer program. This system was originally developed to detect structural lesions and only later applied to the study of intestinal motor function. In the normal, unprepared gut, the existence of gas or transparent liquid content permits a clear view of the intestinal walls and lumen analogous to conventional or subaquatic photography. A major advantage of the system is that recordings are taken with minimal distortion of the physiological conditions in the gut.

Simple observation of the capsule images cannot provide a quantitative estimate of motor activity. Computerised analysis of the endoluminal images has been developed to measure intestinal contractile and non-contractile activity using computer vision and machine learning techniques. Based on these measurements, classification algorithms are then applied to determine whether a case is inside or outside the normal range (previously described in healthy individuals). Cases with abnormal motility are further characterised depending on whether their motor behaviour is hypo- or hyperdynamic [11].

Evaluation of small bowel motility by capsule endoscopy is relatively non-invasive and simple to perform. The analysis is automated, does not require any experience and is bias-free. Initial data indicate the validity of this method for the detection of small bowel dysmotility [12].
Abdominal imaging There are studies that have employed an automated technique to measure the volume of gas in the gut based on analysis of computed tomography (CT) images. A proportion of patients with intestinal neuropathy develop intraluminal gas retention and this may serve to diagnose the dysfunction $[13,14]$.

\section{Involvement of gastrointestinal sensory pathways}

As described above, diabetic neuropathy may affect the neural pathways that control the function, particularly motility, of the stomach and the intestine. Digestive neuropathy in diabetes may also affect the sensory nerves of the gut. Perception of gut signals depends on the stimulation of receptors in the gut wall that activate afferent sensory pathways which conduct signals to the brain cortex. The afferent input can be modulated (either magnified or tuned down), at various levels between the gut wall and the brain. Depending on the pathways affected by the neuropathy, perception may be increased, resulting in a state of hypersensitivity, where stimuli that are normally unperceived induce pain or other gut symptoms. Conversely, gut neuropathy may reduce perception, inducing hyposensitivity [15]. Indeed, studies on gut sensitivity in diabetes characteristically yield variable, even conflicting, results $[9,16]$. It should be noted that some patients develop complete visceral anaesthesia, and therefore have few or no symptoms associated with acute abdominal conditions, such as diverticulitis.

\section{Sensitivity tests}

Gastrointestinal sensitivity can be assessed using probing stimuli that activate afferent sensory pathways [17]. Historically, this has been performed using balloon distension tests. Hollow viscera contain tension receptors that are sensitive to distension and produce conscious perception. Using a simple balloon inflated with a syringe or with more sophisticated methods, such as the barostat or tensostat, gastrointestinal sensation can be measured at any point in the gut [18]. The main drawback of these tests is the absence of standardised thresholds for the diagnosis of hyperor hyposensitivity.

Alternative methods for measuring gut sensory function have been used for research purposes. For example, transmucosal electric nerve stimulation induces sensations similar to those induced by distension. Chemical and thermal stimuli have also been used in the gut to test visceral sensation [15]. Differential stimulation by a combination of techniques may allow more complete characterisation of the sensory dysfunction in patients with diabetic neuropathy. 


\section{Conclusion}

In patients with diabetic neuropathy, disorders of gut motor function may be associated with sensory abnormalities, and the combination of neural pathways affected determines the clinical consequences, and therefore the management. Functional gut symptoms, i.e. symptoms in the absence of structural gut lesions, are very frequent and may affect up to $30 \%$ of the general population with various degrees of severity. However, when these symptoms arise in patients with diabetes, a neuropathy of the gut should be considered and in severe cases should be investigated.

Acknowledgements The authors thank Gloria Santaliestra, Centro de Investigación Biomédica en Red de Enfermedades Hepáticas y Digestivas (Ciberehd) for secretarial assistance.

Funding This work was supported in part by the Spanish Ministry of Economy and Competitiveness (Dirección General de Investigación Cientifica y Técnica, SAF 2013-43677-R). Ciberehd is funded by the Instituto de Salud Carlos III.

Duality of interest The authors have received a research grant from Given Imaging.

Contribution statement Both authors were responsible for drafting the article and revising it critically for important intellectual content. Both authors approved the version to be published.

\section{References}

1. Yarandi SS, Srinivasan S (2014) Diabetic gastrointestinal motility disorders and the role of enteric nervous system: current status and future directions. Neurogastroenterol Motil 26:611-624

2. Schemann M, Reiche D, Michel K (2001) Enteric pathways in the stomach. Anat Rec 262:47-57
3. Bharucha AE, Batey-Schaefer B, Cleary PA et al (2015) Delayed gastric emptying is associated with early and long-term hyperglycemia in type 1 diabetes mellitus. Gastroenterology 149:330-339

4. Chandrasekharan B, Srinivasan S (2007) Diabetes and the enteric nervous system. Neurogastroenterol Motil 19:951-960

5. Abell TL, Camilleri M, Donohoe K et al (2008) Consensus recommendations for gastric emptying scintigraphy: a joint report of the American Neurogastroenterology and Motility Society and the Society of Nuclear Medicine. J Nucl Med Technol 36:44-54

6. Bruno G, Lopetuso LR, Ianiro G et al $(2013){ }^{13} \mathrm{C}$-Octanoic acid breath test to study gastric emptying time. Eur Rev Med Pharmacol Sci 17(Suppl 2):59-64

7. Muresan C, Surdea Blaga T, Muresan L et al (2015) Abdominal ultrasound for the evaluation of gastric emptying revisited. J Gastrointestin Liver Dis 24:329-338

8. Azpiroz F, Feinle-Bisset C, Grundy D et al (2014) Gastric sensitivity and reflexes: basic mechanisms underlying clinical problems. J Gastroenterol 49:206-218

9. Kumar A, Attaluri A, Hashmi S et al (2008) Visceral hypersensitivity and impaired accommodation in refractory diabetic gastroparesis. Neurogastroenterol Motil 20:635-642

10. Stanghellini V, Camilleri M, Malagelada JR (1987) Chronic idiopathic intestinal pseudo-obstruction: clinical and intestinal manometric findings. Gut 28:5-12

11. Malagelada C, Drozdzal M, Seguí S et al (2015) Classification of functional bowel disorders by objective physiological criteria based on endoluminal image analysis. Am J Physiol Gastrointest Liver Physiol 309:G413-G419

12. Malagelada C, De Iorio F, Azpiroz F et al (2008) New insight into intestinal motor function via noninvasive endoluminal image analysis. Gastroenterology 135:1155-1162

13. Bendezú RA, Barba E, Burri E et al (2015) Intestinal gas content and distribution in health and in patients with functional gut symptoms. Neurogastroenterol Motil 27:1249-1257

14. Serra J, Villoria A, Azpiroz F et al (2010) Impaired intestinal gas propulsion in manometrically proven dysmotility and in irritable bowel syndrome. Neurogastroenterol Motil 22:401-402

15. Frøkjaer JB, Andersen SD, Ejskaer N et al (2007) Gut sensations in diabetic autonomic neuropathy. Pain 131:320-329

16. Søfteland E, Brock C, Frøkjaer JB et al (2014) Rectal sensitivity in diabetes patients with symptoms of gastroparesis. J Diabetes Res 2014:784841

17. Azpiroz F (2002) Gastrointestinal perception: pathophysiological implications. Neurogastroenterol Motil 14:229-239

18. Distrutti E, Azpiroz F, Soldevilla A et al (1999) Gastric wall tension determines perception of gastric distention. Gastroenterology 116: $1035-1042$ 